\title{
Relación entre clima organizacional y riesgo psicosocial intralaboral de una empresa de transporte en Bucaramanga
}

Relationship between organizational climate and intra-labor psychosocial risk of a transport company in Bucaramanga

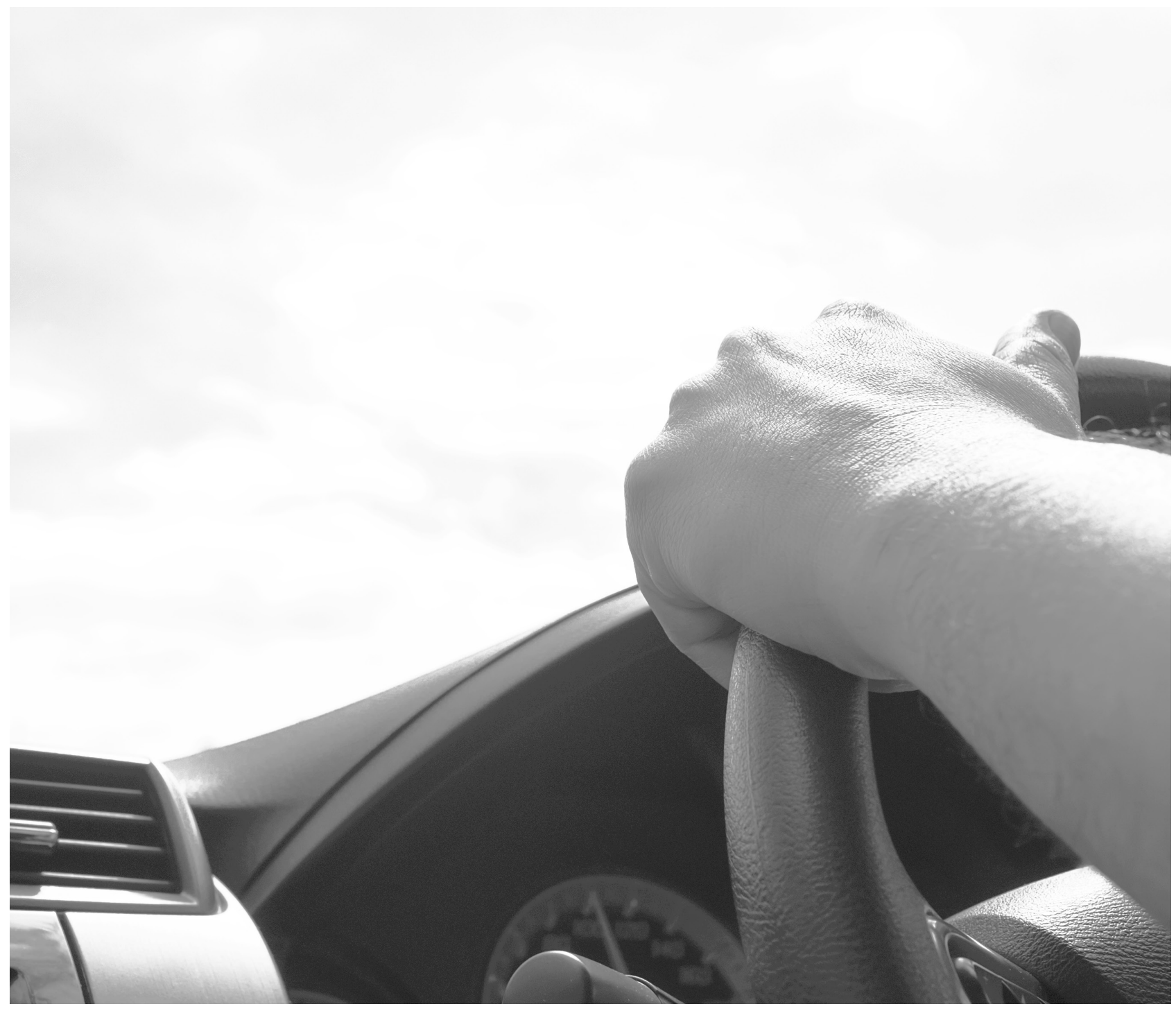




\title{
Relación entre clima organizacional y riesgo psicosocial intralaboral de una empresa de transporte en Bucaramanga ${ }^{1}$ Relationship between organizational climate and intra-labor psychosocial risk of a transport company in Bucaramanga
}

\author{
Sebastián Garcia-Mendez², Yurley Paola Galvis-Rojas³ , Jhonatan Andrés Ortiz-Olago ${ }^{4}$
}

Artículo recibido en marzo 28 de 2019; artículo aceptado en octubre 19 de 2019

Este artículo puede compartirse bajo la Licencia Creative Commons Atribución-NoComercial-Compartir lgual 4.0 Internacional y se referencia usando el siguiente formato: Garcia-Mendez, S., Galvis-Rojas, Y. P. \& Ortiz-Olago, J.A. (2020). Relación entre clima organizacional y riesgo psicosocial intralaboral de una empresa de transporte en Bucaramanga.

I+D Revista de Investigaciones, 15 (1), 67-75. DOI: https://doi.org/10.33304/revinv.v15n1-2020007

\section{Resumen}

Este documento muestra la relación entre clima organizacional y el nivel de riesgo psicosocial intralaboral en empleados operativos de la empresa de transporte Cotaxi S. A. S., de Bucaramanga. Para el desarrollo de esta investigación se determina el clima organizacional en las dimensiones objetivos, cooperación, liderazgo, toma de decisiones, relaciones interpersonales, motivación y control, mediante la aplicación del instrumento IMCOC; seguido del nivel de riesgo psicosocial en los dominios liderazgo y relaciones sociales en el trabajo, control sobre el trabajo, demanda de trabajo y recompensas, aplicando la batería propuesta por el Ministerio de Protección Social, para finalmente determinar su relación mediante la correlación de Pearson. Los hallazgos de este estudio indican que una empresa con un clima organizacional que posee una buena cooperación entre los miembros de la organización, un control adecuado sobre las tareas y alta satisfacción laboral tiene niveles de riesgos psicosociales intralaborales bajos concernientes principalmente con las relaciones interpersonales.

Palabras clave: Clima organizacional, riesgo psicosocial, correlación, sector transporte, Colombia.

\begin{abstract}
This document shows the relationship between organizational climate and level of intra-labor psychosocial risk in operative employees of the transport company Cotaxi S. A. S. of Bucaramanga. For the development of this research, the organizational climate is determined in the objectives, cooperation, leadership, decision-making, interpersonal

\footnotetext{
' Artículo de investigación de tipo correlacional, de corte transversal, con enfoque mixto (cuantitativo y cualitativo), resultado de un proyecto de investigación culminado, perteneciente al área de dirección empresarial, subárea de comportamiento organizacional y gestión de recursos humanos, desarrollado en la Universidad de Investigación y Desarrollo (Bucaramanga, Colombia). Dirección: calle 9 n. ${ }^{\circ} 23-55$, PBX: 6352525. Fecha de inicio: febrero de 2018. Fecha de terminación: noviembre de 2018.

${ }^{2}$ Magíster en Administración. Grupo de Investigación Sinergia, profesor de la Universidad de Investigación y Desarrollo (Bucaramanga, Colombia). Dirección: calle 9 n. ${ }^{\circ}$ 23-55, PBX: 6352525. ORCID ID: https://orcid.org/0000-0002-6077-0248. Correo electrónico institucional: sgarcia15@udi.edu.co.

${ }^{3}$ Ingeniera industrial. Universidad de Investigación y Desarrollo. Actualmente vinculada al Servicio Nacional de Aprendizaje (Bucaramanga, Colombia). Dirección: calle 15 n. ${ }^{\circ}$ 27-37, PBX: 6800600. ORCID ID: https://orcid.org/0000-0001-7713-3213. Correo electrónico: galvisyurley@hotmail.es.

${ }^{4}$ Ingeniero industrial. Universidad de Investigación y Desarrollo (Bucaramanga, Colombia). Dirección: calle 9 n. ${ }^{\circ} 23-55$, PBX: 6352525. ORCID ID: https://orcid.org/0000-0002-0612-4480. Correo electrónico: jhonaandres@hotmail.com.
} 
relations, motivation, and control dimensions, through the application of the IMCOC instrument. This is followed by the level of psychosocial risk in the leadership and social relations at work, control over work, and demand for work and rewards domains, applying the battery proposed by the Ministry of Social Protection, to finally determine their relationship through the Pearson correlation. The findings of this study indicate that a company with an organizational climate that has a good cooperation among the members of the organization, adequate control over the tasks to be performed, and high job satisfaction has low intra-labor psychosocial risks, concerning mainly interpersonal relationships.

Keywords: Organizational climate, psychosocial risk, correlation, transport sector, Colombia.

\section{Introducción}

Las organizaciones están conformadas e integradas por seres humanos que cumplen los objetivos de la empresa, en relación con su objeto o razón social. Estos van a ser determinados por los tipos de relaciones que se establezcan entre ellos de manera intralaboral y, a su vez, los riesgos que se generen con base en estas relaciones. En este orden de ideas, el capital humano, en su constante interacción, constituye un factor determinante que puede influir positiva o negativamente en el cumplimiento de los objetivos empresariales.

Hablar del clima organizacional hoy en las empresas se convierte en una prioridad, ya que este es determinante al momento de cumplir los objetivos y, a su vez, crear un plus en la organización para ser más competente en el mercado en que se desenvuelve. Es importante para cualquier organización tener claras y además crear o establecer acciones que les permitan mitigar riesgos que, desde lo psicosocial, fatiguen al empleado, debido a que los riesgos se ven asociados a las condiciones que se encuentran presentes en una situación laboral y que están directamente relacionadas con el entorno ambiental con respecto a la organización, los procedimientos, los métodos y los contenidos de trabajo, la realización de las tareas, la carga laboral, la capacidad de control sobre la propia tarea y la supervisión. Estos aspectos afectan la calidad de vida del trabajador y la calidad de su desempeño (Salazar Estrada, Guerrero Pupo, Machado Rodríguez \& Cañedo Andalia, 2009).

En cuanto a esto, en el mundo del transporte, tanto de pasajeros como de carga, se identifican algunos factores externos e internos que están en una constante fluctuación, de modo que generan climas de mucha tensión y riesgo para todos los que hacen parte de estas organizaciones, así como para los clientes de dichos servicios. Estos factores constituyen un alto riesgo psicosocial, por lo que las empresas de este sector deben reconocer y evaluar el contexto en el cual se desempeña su personal o sus colaboradores, ya que el óptimo desarrollo de estas empresas es importante para la región (Leaño, 2018).

Actualmente, existen diferentes herramientas que le permiten a las organizaciones, desde el área de recursos humanos, evaluar el clima laboral y los riesgos psicosociales intralaborales, con el fin de generar acciones que posibiliten la mejora y mitigación de estos (Reinoso Alarcón \& Araneda Cea, 2007).

Con base en lo anterior, el presente documento intenta analizar la relación existente entre el clima organizacional y los niveles de riesgos psicosociales intralaborales en el personal operativo de una empresa del sector transporte de carga terrestre de Bucaramanga, específicamente de la empresa Cotaxi S. A. S., que cuenta con 57 años de experiencia en el mercado. Este análisis se desarrolla por medio de la aplicación de la encuesta IMCOC (Instrumento para Medir Clima en Organizaciones Colombianas) y la batería propuesta por el Ministerio de Protección Social, mediante el análisis de correlación de Pearson.

\section{Clima organizacional}

De acuerdo con las definiciones brindadas por diferentes autores (Cornell, 1955; Hall \& Deras Quiñones, 1996; Méndez, 2006; Palacios, 1998; Rodríguez, 2001 y Seisdedos, 1996), el 'clima organizacional' se define como el conjunto de percepciones globales que comparten los miembros de una organización respecto al trabajo, el ambiente físico en que esta se da, las relaciones interpersonales que tienen lugar en torno a él, las diversas regulaciones formales que afectan a dicho trabajo, que orientan su actitud, grado de participación e influyen de forma directa o indirecta en la motivación de los empleados, y determinan su comportamiento, satisfacción y nivel de eficiencia en el trabajo (Brunet, 1987; Méndez, 2006).

Otros autores, como Gibson, Donnelly y Ivancevich (1991), expresaron que el clima organizacional constituye el último elemento para describir la estructura psicológica de las organizaciones. El clima es la "sensación", "personalidad" o "carácter" del ambiente de la empresa. En tal sentido, indicaron que está determinado por el comportamiento de las personas a nivel individual, grupal, de liderazgo, la estructura de la organización y los procesos organizacionales. Por tanto, los resultados o las consecuencias del clima se establecen con base en los rendimientos individuales, grupales y organizacionales. 
En el marco del presente documento, se utilizará la definición de clima organizacional de los autores Litwin y Stringer (1968), que permite entender el clima organizacional como una característica relativamente permanente del entorno interno de la organización que es experimentado por sus miembros e influye en el comportamiento. Litwin y Stringer (1968) son pioneros en la medición del clima laboral y postulan la existencia de nueve dimensiones que describen el clima existente en determinada empresa. A continuación, se presentan dichas dimensiones.

\section{Dimensiones del clima organizacional}

Las dimensiones del clima organizacional son los factores que influyen en el comportamiento de los individuos, que pueden originarse con base en distintas variables de la organización (Torrecilla, 2005). Varios autores han propuesto definir la cantidad y naturaleza de dichos factores, entre los que se destacan Litwin y Stringer, (1968), quienes, a partir de estos, postulan las siguientes nueve dimensiones:

- Estructura: Hace referencia a las reglas, normas, procedimientos, trámites, obstáculos y otras limitaciones a las que se ven enfrentados los trabajadores en el desempeño de su labor.

- Responsabilidad: Es la percepción de los miembros de la organización acerca de la autonomía en la toma de decisiones relacionadas al trabajo.

- Recompensa: Corresponde a la percepción sobre el premio o incentivo recibido por el trabajo bien hecho.

- Desafío: Hace referencia a las metas que los trabajadores tienen respecto a determinados riesgos que pueden sufrir durante la realización de sus tareas.

- Relaciones: Percepción del ambiente grato de trabajo y de las buenas relaciones sociales dentro y fuera de la organización.

- Cooperación: Sentido sobre el espíritu de ayuda y apoyo mutuo de parte de los directivos y demás empleados.

- Estándares: Se refiere a la percepción de los miembros de la empresa acerca de los niveles de rendimiento y productividad que se han fijado en la organización.

- Identidad: Sentimiento de pertenencia a la compañía.

Adicionalmente, otros autores, como Likert (1968), llegan a tipificar cuatro tipos de sistemas organizacionales, cada uno con un clima particular: el autoritario, caracterizado por la desconfianza, ya que sigue una línea altamente burocratizada de conducto regular; el paternal, con autoridades que tienen todo el poder pero que conceden ciertas facilidades a sus subordinados; el consultivo, con mayor grado de descentralización y delegación de funciones, y el participativo, en el cual las decisiones especificas son adoptadas por escalones medios e inferiores.

En este contexto los sistemas organizacionales analizan el clima laboral de diferentes maneras, partiendo por su grado de confianza o desconfianza, responsabilidad o temor e inseguridad que se pueden identificar dentro de la empresa.

\section{Estrategia de medición del clima organizacional}

A partir del modelo teórico de las relaciones humanas y las variables identificadas por Likert (1968), se han desarrollado varios instrumentos de medición para el clima organizacional. Por ejemplo, actualmente en Colombia existe un instrumento para realizar esta medición en las empresas, Ilamado IMCOC (Instrumento para Medir Clima en Organizaciones Colombianas), realizado con el objetivo de brindar mejoras significativas en los indicadores de gestión del factor humano. Dicho instrumento fue desarrollado por Méndez (2006), profesor de Administración de la Universidad del Rosario, luego de realizar diversas investigaciones desde 1980 hasta 2005.

Méndez (2006) menciona que el instrumento posibilita el conocimiento de la variable objeto de análisis, con el propósito de establecer programas de acción. Las variables utilizadas para la medición del clima organizacional por el modelo IMCOC se describen a continuación:

- Objetivos: Su enfoque se basa en el conocimiento que el trabajador tiene sobre la razón de ser y los fines hacia los cuales se orienta la empresa en la que trabaja; esto gracias a la satisfacción de las necesidades y sentido de pertenencia con la empresa.

- Cooperación: Procesos asociativos entre los miembros de la empresa, siempre y cuando estén en el ejercicio de su función, lo que permite el cumplimiento de los objetivos organizacionales propuestos.

- Liderazgo: Persona en la organización que ejerce su acción con el uso de elementos y comportamientos que el marco de la teoría administrativa define como forma o estilo de dirección. 
- Toma de decisiones: Subproceso de la función de dirección, relacionado con el estilo de dirección que ejerza el líder en la organización.

- Relaciones interpersonales: Interacción y desarrollo de relaciones sociales de manera asociativa.

- Motivación: Satisfacción laboral, percepción del objetivo de liderazgo y actitudes en sus actividades diarias.

- Control: Identifica si el trabajo realizado en un tiempo determinado ha permitido el cumplimiento de los objetivos corporativos (periodicidad) con los que se realiza una función.

Estas variables planteadas por el instrumento IMCOC permiten determinar cómo se encuentra el clima organizacional dentro de una empresa $y$, por ende, tomar las medidas pertinentes para mejorar o mantener un buen clima, y de esta manera la empresa puede tener una mejor productividad y buenas relaciones internas.

\section{Riesgos psicosociales intralaborales}

Los riesgos psicosociales intralaborales son las condiciones presentes en el entorno de trabajo que están relacionadas con la organización, las características propias del puesto de trabajo y la tarea a realizar, que tienden a afectar el desarrollo normal del trabajo y la salud de los empleados de una organización (Rosero, Álvarez, 2012). Pueden provocar problemas cognitivos, emocionales y conductuales que pueden derivar en problemas de salud física y mental.

El Ministerio de Salud y Protección Social define estos riesgos como las interacciones entre el trabajador, el medioambiente y la organización empresarial, ligados al proceso de producción, a las modalidades de la gestión administrativa que pueden generar una carga psicológica, fatiga mental, alteraciones de la conducta, del comportamiento y reacciones fisiológicas. Por otra parte, el Comité Mixto OIT-OMS sobre Medicina del Trabajo (1984) indicó que los factores de riesgo psicosocial en el trabajo abarcan las interacciones entre el medioambiente laboral, las características de las condiciones de trabajo, las relaciones entre los trabajadores, la organización, las características del trabajador, la cultura, las necesidades y la situación personal fuera del trabajo.

\section{Principales riesgos psicosociales}

De acuerdo con lo anterior, por causa de dichos factores, los trabajadores de las organizaciones se ven expuestos a diferentes riesgos psicosociales. Entre ellos, los principales son estrés, violencia, acoso laboral, acoso sexual e inseguridad contractual. El estrés laboral, de acuerdo con la Comisión Europea, se define como:

El patrón de reacciones emocionales, cognitivas, fisiológicas y de comportamiento a ciertos aspectos adversos o nocivos del contenido del trabajo, organización del trabajo y el medioambiente de trabajo, que se caracteriza por altos niveles de excitación y de respuesta y la frecuente sensación de no poder afrontarlos (Comisión Europea, 2002).

Por su parte, la OIT define la violencia laboral como "acción, incidente o comportamiento que se aparta de lo razonable, en la cual una persona es asaltada, amenazada, humillada o lesionada como consecuencia directa de su trabajo" (OIT, 2003). En cuanto al acoso laboral, el Comité Consultivo de la Comisión Europea para la Seguridad, la Higiene y la Protección de la Salud en el Trabajo refiere el siguiente concepto:

Forma de comportamiento negativo entre compañeros o entre superiores jerárquicos y subordinados, por el que la persona en cuestión es humillada y atacada varias veces, directa o indirectamente por una o más personas con el propósito y con el efecto de incomodarla (Alegría, 2011).

Asimismo, la Jurisprudencia Estadounidense considera el acoso sexual en el trabajo como:

Toda conducta de naturaleza sexual desarrollada en el ámbito de organización y dirección de una empresa, o en relación, o como consecuencia de una relación de trabajo, realizado por un sujeto que sabe o debe saber que es ofensiva y no deseada por la víctima, determinando una situación que afecta al empleo y a las condiciones de trabajo creando un entorno laboral ofensivo, hostil, intimidatorio o humillante (Jiménez \& León, 2010).

Por último, la inseguridad contractual hace referencia a la preocupación general acerca de la existencia del trabajo en el futuro, y también como una amenaza percibida de características de trabajo diferentes, tales como la posición dentro de una organización o las oportunidades de carrera.

En el plano nacional, se resalta la norma GTC 45 como una guía orientadora para identificar los peligros y valorar los riesgos en torno a la seguridad industrial, que relaciona el siguiente proceso: definir instrumento y recolectar información; clasificar los procesos, las actividades y las tareas; identificar los peligros; identificar los controles existentes; evaluar el riesgo; definir los criterios para determinar la aceptabilidad del riesgo; definir la aceptabilidad del riesgo; elaborar el plan de acción; revisar la conveniencia del plan de acción; mantener y 
actualizar y documentar (Icontec, 2010). Asimismo, se resalta la presencia de la Ley 1010 de 2006 concerniente a los tipos de acoso laboral: maltrato laboral, persecución laboral, discriminación laboral, entorpecimiento laboral, inequidad laboral y desprotección laboral (Gobierno de Colombia, 2006).

\section{Métodos de evaluación}

Los métodos de evaluación de los riesgos psicosociales son muy diversos, tales como la evaluación conductual por medio del modelo de los autores Fellner y SulzarAzaroff (1984); el método de evaluación de factores psicosociales propuesto por el Instituto Nacional de Seguridad e Higiene en el Trabajo; el cuestionario psicosocial emitido por Copenhague; el cuestionario de factores psicosociales en el trabajo diseñado por Espinoza y Romero (2002), entre otros (Uribe, 2013).

En el marco de la legislación colombiana, se destaca el Cuestionario de factores de riesgo psicosocial intralaboral forma A y B, creado por el Ministerio de Protección Social, que consta de dos formas de aplicación: la forma $A$, destinada a jefes profesionales o técnicos; y la forma $B$, dirigida a auxiliares $u$ operarios. En ese orden de ideas, se valida la idoneidad para la presente investigación teniendo en cuenta la alineación con los entes gubernamentales. La forma B evalúa los dominios demandas del trabajo, control, liderazgo, relaciones sociales en el trabajo y recompensa.

\section{Correlación de Pearson}

Según Martínez Cortés y Pérez (2016), quienes citan a Cortés-Reyes, Rubio-Romero y Gaitán-Duarte (2010), existen diferentes investigaciones que utilizan el coeficiente de correlación de Pearson para establecer una ecuación lineal entre dos variables; en otros términos, un cambio en una unidad de las variables genera un cambio en la otra variable. Los valores de la correlación de Pearson se enmarcan entre 0 y 1 .

\section{Cotaxi S. A. S.}

Empresa de carácter cooperativo, fundada el 31 de octubre de 1961, cuyo objeto social es el transporte de pasajeros y carga, con presencia en diversos departamentos en el ámbito nacional, tales como Santander, Norte de Santander, Cesar, Magdalena, Caldas, Boyacá, Tolima y Bolívar. Surgió como una iniciativa de taxistas en el marco de brindar un servicio diferenciador para los clientes (Cotaxi, 2018).

\section{Metodología}

\section{Tipo de estudio}

El presente estudio se consolidó bajo el enfoque mixto, con el fin de recolectar, analizar y vincular datos cuantitativos y cualitativos (Gómez, 2015), para responder al planteamiento entre las diferentes variables objeto de estudio: clima organizacional y nivel de riesgo psicosocial intralaboral. Por su parte, la investigación fue de tipo de correlacional y transversal, ya que implica la sistematización de las relaciones entre las variables de una muestra específica para ser analizada estadísticamente.

\section{Procedimiento}

Para el presente estudio se midió la percepción del clima organizacional de los trabajadores operativos de la empresa Cotaxi S. A. S. utilizando la encuesta IMCOC. Posteriormente se evaluaron los riesgos psicosociales intralaboral forma B. Finalmente, para determinar la relación existente entre las variables, se analizó el coeficiente de correlación de Pearson en las diversas dimensiones o dominios.

\section{Participantes}

En el presente estudio participaron 100 empleados operativos de la empresa Cotaxi S. A. S. El $86 \%$ de los trabajadores que hicieron parte de esta investigación son hombres. En cuanto al estado civil de los encuestados, el $42 \%$ son casados; el $30 \%$, solteros, y el $18 \%$ se encuentran en unión libre. Además, el principal nivel de escolaridad es bachillerato $(84 \%)$, mientras que en menor medida los empleados son técnicos (12\%), profesionales (3\%) o tecnólogos (1\%).

\section{Materiales e instrumentos}

Para medir el clima organizacional, se utiliza la encuesta IMCOC, desarrollada en 1981 por la Universidad del Rosario. Esta prueba se encuentra fundamentada en el modelo teórico de las relaciones humanas y las variables identificadas por el instrumento de Likert. Asimismo, este instrumento se encuentra diseñado en forma de cuestionario y debe ser resuelto por cada uno de los trabajadores, de tal forma que la opinión personal contribuye en la obtención de resultados grupales, que estadísticamente permiten establecer la situación actual de la empresa (Méndez, 2006).

En relación con la evaluación de los riesgos psicosociales, se utiliza el Cuestionario de factores de riesgo psicosocial intralaboral forma B, establecido por el Ministerio de Salud y Protección Social de Colombia. Dicho instrumento permite identificar y evaluar los riesgos psicosociales. Es uno de los más utilizados en los últimos años. En este caso, se aplicará la forma B, dadas las características de la población objeto de estudio, ya que la forma B está dirigida al personal operativo (Charria, Sarsosa \& Arenas, 2011). 


\section{Resultados}

Los resultados objeto de presentación relacionan el clima organizacional, la evaluación de los niveles de riesgo psicosociales intralaborales y finalmente los coeficientes de correlación de Pearson para las variables de la empresa Cotaxi S. A. S.

\section{Clima organizacional}

El clima organizacional en la empresa Cotaxi S. A. S. a nivel general es un clima favorable, debido principalmente a los instrumentos motivacionales y a los procesos de control entre las instancias organizacionales (ver Tabla 1). Al examinar el clima laboral por unidad de análisis, se observa que no existe variabilidad en la percepción del clima laboral por parte de estos, ya que consideran que la empresa objeto de estudio tiene un clima laboral favorable, o muy favorable por parte de los empleados con un nivel de formación tecnóloga (ver Tabla 2). Dichos patrones fueron determinados con base en el instrumento de recolección de la información aplicado.

Tabla 1

Clima organizacional

\begin{tabular}{ccc}
\hline Dimensiones & Puntaje & Nivel del clima \\
\hline Objetivos & 75,8 & Favorable \\
Cooperación & 69,6 & Medianamente favorable \\
Liderazgo & 77,5 & Favorable \\
Toma de decisiones & 61,9 & Medianamente favorable \\
Relaciones & 77,2 & Favorable \\
interpersonales & 81,7 & Favorable \\
Motivación & 79,6 & Favorable \\
Control & 75,1 & Favorable \\
Total & & \\
\hline
\end{tabular}

Fuente: Autores.

Tabla 2

Clima organizacional por unidad de análisis

\begin{tabular}{ccc}
\hline Unidad de análisis & Puntaje & Nivel del clima \\
\hline Género masculino & 75,6 & Favorable \\
Género femenino & 72,4 & Favorable \\
Estado civil casado(a) & 74,9 & Favorable \\
Estado civil soltero(a) & 75,6 & Favorable \\
Estado civil unión libre & 74,9 & Favorable \\
Profesional & 77,1 & Favorable \\
Tecnólogo & 90,5 & Muy favorable \\
Técnico & 71,9 & Favorable \\
Bachiller & 75,3 & Favorable \\
\hline
\end{tabular}

Fuente: Autores.

\section{Riesgos psicosociales intralaborales}

En relación con el nivel de riesgo psicosocial intralaboral se observa que este es alto, y el dominio con el mayor nivel de riesgo es el que hace referencia a las demandas de trabajo de tipo ambiental, físico, emocional, cuantitativo, extralaboral y mental (ver Tabla 3 ).

Tabla 3

Niveles de riesgos psicosociales intralaborales

\begin{tabular}{ccc}
\hline Dominios & Puntaje & Nivel del riesgo \\
\hline Liderazgo y relaciones & 28,6 & Alto \\
sociales en el trabajo & 35,8 & Alto \\
Control sobre el trabajo & 36,4 & Muy alto \\
Demandas de trabajo & $46,4,8$ & Alto \\
Recompensas & 25,8 & Alto \\
Total & 36,8 &
\end{tabular}

Fuente: Autores.

En cuanto a los riesgos psicosociales obtenidos por unidad de análisis, se observa que la mano de obra femenina de la organización tiene niveles de riesgo muy altos, debido principalmente a las cargas de trabajo y a las recompensas recibidas por su labor. Mientras que los trabajadores que han realizado una tecnología presentan un nivel de riesgo psicosocial bajo derivado principalmente por la claridad del rol que desempeñan dentro de la empresa, la capacitación recibida (Argüello-López, Uribe-Bermúdez \& ValdiviesoGuerrero, 2017) y las bajas demandas de trabajo mental y físico (ver Tabla 4).

Tabla 4

Niveles de riesgos psicosociales intralaborales por unidad de análisis

\begin{tabular}{ccc}
\hline Unidad de análisis & Puntaje & Nivel del riesgo \\
\hline Género masculino & 36,1 & Alto \\
Género femenino & 40,9 & Muy alto \\
Estado civil casado(a) & 36,1 & Alto \\
Estado civil soltero(a) & 37,6 & Alto \\
Estado civil unión libre & 30,7 & Medio \\
Profesional & 36,3 & Alto \\
Tecnólogo & 22,7 & Bajo \\
Técnico & 35,8 & Alto \\
Bachiller & 37,1 & Alto \\
\hline
\end{tabular}

Fuente: Autores.

\section{Correlación de Pearson}

Con el propósito de encontrar la relación existente entre las dimensiones del clima organizacional y los dominios de los riesgos psicosociales intralaborales, se hallan los coeficientes de Correlación de Pearson. En la Tabla 5 se observa el grado de asociación entre las variables. Estos coeficientes de correlación se encuentran entre -0,695 y 0,212 .

Al analizarlos, se evidencia que las mayores relaciones se presentan entre cooperación y relaciones sociales en el trabajo $(-0,60)$, control sobre el trabajo y liderazgo $(-0,59)$, motivación o satisfacción laboral y reconocimiento $(-0,57)$. Dichas relaciones son altas y significativas, 
pero de magnitud negativa, lo que significa que, a medida que aumentan los procesos asociativos entre los miembros de la empresa, el control sobre el trabajo - la motivación laboral disminuyen respectivamente los riesgos psicosociales en las relaciones sociales en el trabajo, el liderazgo o las recompensas. Por tanto, una empresa con un clima organizacional que posee una buena cooperación entre los miembros de la organización, un control adecuado sobre las tareas a realizar y alta satisfacción laboral tiene niveles de riesgos psicosociales intralaborales bajos, principalmente en aspectos relacionados con las relaciones interpersonales entre los miembros de la organización, liderazgo y reconocimiento (Orellana \& Portalanza, 2014).

Tabla 5

Correlación de Pearson entre las variables del clima organizacional y los riesgos psicosociales

\begin{tabular}{|c|c|c|c|c|c|c|c|c|c|c|c|c|c|c|c|c|}
\hline & 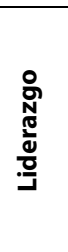 & 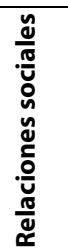 & 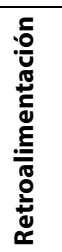 & $\bar{\alpha}$ & 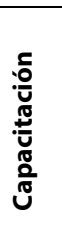 & 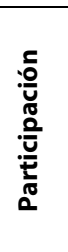 & 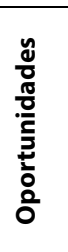 & 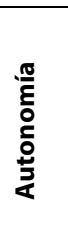 & 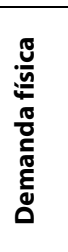 & 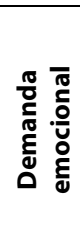 & 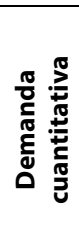 & 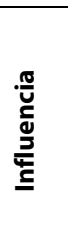 & 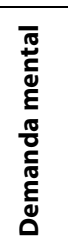 & 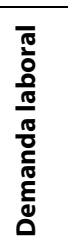 & 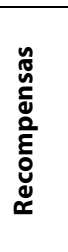 & 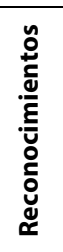 \\
\hline Objetivos & $\begin{array}{c}- \\
0,41\end{array}$ & $\begin{array}{c}\overline{-} \\
0,49\end{array}$ & $\begin{array}{c}- \\
0,43\end{array}$ & $\begin{array}{c}- \\
0,36\end{array}$ & $\begin{array}{c}- \\
0,44\end{array}$ & 0,29 & $\begin{array}{c}- \\
0,31\end{array}$ & $\begin{array}{c}- \\
0,23\end{array}$ & $\begin{array}{c}- \\
0,36\end{array}$ & 0,03 & $\begin{array}{c}- \\
0,21\end{array}$ & $\overline{-}$ & 0,20 & $\begin{array}{c}- \\
0,05\end{array}$ & $\begin{array}{c}- \\
0,55\end{array}$ & $\begin{array}{c}- \\
0,52\end{array}$ \\
\hline Cooperación & 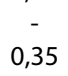 & $\begin{array}{c}- \\
0,60\end{array}$ & $\begin{array}{c}- \\
0,50\end{array}$ & $\begin{array}{c}- \\
0,30\end{array}$ & $\begin{array}{c}- \\
0,22\end{array}$ & 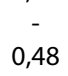 & 0,40 & $\begin{array}{c}- \\
0,40\end{array}$ & $\begin{array}{c}- \\
0,29\end{array}$ & 0,03 & $\begin{array}{c}- \\
0,34\end{array}$ & $\begin{array}{c}- \\
0,10\end{array}$ & 0,04 & $0,-$ & $0,-$ & - \\
\hline Liderazgo & $\begin{array}{c}- \\
0,65\end{array}$ & $\begin{array}{c}- \\
0,53\end{array}$ & $\begin{array}{c}- \\
0,34\end{array}$ & $\begin{array}{c}- \\
0,49\end{array}$ & $\begin{array}{c}- \\
0,45\end{array}$ & $0,-$ & 0,31 & $0,-$ & $0,-$ & 0,17 & $0,-$ & $\begin{array}{c}- \\
0,12\end{array}$ & 0,21 & $0,-$ & $\begin{array}{c}- \\
0,53\end{array}$ & - \\
\hline $\begin{array}{c}\text { Toma } \\
\text { decisiones }\end{array}$ & $0,-$ & 0,29 & 0,42 & 0,32 & 0,21 & 0,48 & $\begin{array}{c}- \\
0,31\end{array}$ & $\begin{array}{c}- \\
0,30\end{array}$ & 0,27 & 0,02 & $\begin{array}{c}- \\
0,09\end{array}$ & 0,03 & 0,02 & 0,15 & $\begin{array}{c}- \\
0,33\end{array}$ & 0,48 \\
\hline Relaciones & $\begin{array}{c}- \\
0,54\end{array}$ & $\begin{array}{c}- \\
0,55\end{array}$ & 0,48 & $\begin{array}{c}- \\
0,32\end{array}$ & $\begin{array}{c}- \\
0,24\end{array}$ & $0,-$ & 0,34 & $\begin{array}{c}- \\
0,14\end{array}$ & 0,29 & 0,05 & $\begin{array}{c}- \\
0,21\end{array}$ & $\begin{array}{c}- \\
0,06\end{array}$ & 0,14 & 0,17 & $\begin{array}{c}- \\
0,50\end{array}$ & $\begin{array}{c}- \\
0,50\end{array}$ \\
\hline Motivación & $\begin{array}{c}- \\
0,50\end{array}$ & $\begin{array}{c}- \\
0,50\end{array}$ & $\begin{array}{c}- \\
0,36\end{array}$ & $\begin{array}{c}- \\
0,36\end{array}$ & $\begin{array}{c}- \\
0,36\end{array}$ & $\begin{array}{c}- \\
0,33\end{array}$ & $0,-$ & $\begin{array}{c}- \\
0,17\end{array}$ & - & 0,12 & $\begin{array}{c}- \\
0,15\end{array}$ & $\begin{array}{c}- \\
0,49\end{array}$ & 0,21 & 0,19 & $\begin{array}{c}- \\
0,70\end{array}$ & $\begin{array}{c}- \\
0,57\end{array}$ \\
\hline Control & , & $\begin{array}{c}- \\
0,47\end{array}$ & $\begin{array}{c}- \\
0,51\end{array}$ & $\begin{array}{c}- \\
0,34\end{array}$ & $\begin{array}{c}- \\
0,34\end{array}$ & $\begin{array}{c}- \\
0,48\end{array}$ & $\begin{array}{c}- \\
0,45 \\
0\end{array}$ & $\begin{array}{c}- \\
0,22 \\
\end{array}$ & $0,-$ & 0,01 & $0,-$ & $\begin{array}{c}- \\
0,02\end{array}$ & 0,11 & $\begin{array}{c}- \\
0,27 \\
\end{array}$ & $\begin{array}{c}- \\
0,46\end{array}$ & $\begin{array}{c}- \\
0,52 \\
\end{array}$ \\
\hline
\end{tabular}

Fuente: Autores.

\section{Conclusiones}

El clima organizacionaly losfactores de riesgopsicosocial intralaborales son ejes fundamentales de trabajo en el marco de la salud y el bienestar de los trabajadores. Por tal motivo, la organización objeto de estudio debe tener una visión clara de la importancia de la gestión del personal en el marco de generar programas para los sujetos en mención, con el propósito de forjar vínculos afines al compromiso organizacional, que posibiliten el cumplimiento de los objetivos empresariales en un plano temporal.

Como se aprecia, el clima organizacional es favorable, en gran medida por las dimensiones correspondientes a motivación $(81,7)$, control $(79,6)$ y liderazgo $(77,5)$. En contraste, lo correspondiente a toma de decisiones $(61,9)$ y cooperación $(69,5)$ es aún objeto de mejora. En otros términos, la empresa deberá establecer mecanismos de trabajo conjunto con los empleados operativos, con el fin de generar soluciones integrales generadas por la opinión de las diversas partes interesadas internas de la organización.

Los factores de riesgo psicosociales intralaborales registran niveles de riesgo alto o muy alto. En tal sentido, el dominio correspondiente a demandas de trabajo $(46,4)$ deberá ser analizado al detalle por la organización, con el propósito de establecer con exactitud la capacidad de los empleados en el marco de las exigencias emitidas por la empresa. Las organizaciones tienen metas acotadas a la temporalidad; por tal motivo, deben contar con recurso idóneo en cantidad y calidad para cumplirlas eficientemente.

La relación entre las variables objeto de estudio permite visualizar que las dimensiones de los factores de riesgo psicosocial intralaborales más influenciadas por los dominiosdel climalaboralsoncaracterísticas delliderazgo, relaciones sociales en el trabajo, retroalimentación del desempeño, claridad del rol, capacitación, participación, manejo del cambio y reconocimiento. Por consiguiente, se deberán generar estrategias enfocadas a la reacción en cadena del mejoramiento de la gestión del personal.

\section{Recomendaciones}

Para la empresa objeto de estudio, Cotaxi S. A. S., se recomienda definir e implementar diferentes estrategias para mitigar los niveles altos de riesgos psicosociales 
intralaborales encontrados en este estudio y para mejorar o mantener el clima organizacional favorable.

También sería interesante replicar este análisis en otras empresas, utilizando otras formas para medir el clima organizacional o los riesgos psicosociales intralaborales y considerando otras variables que pueden influenciar sobre estos factores, como la ubicación de la empresa, el sector al que pertenece, los estratos socioeconómicos y el tipo de contratación de los empleados.

\section{Referencias}

Alegría, C. A. (2011). Aproximación conceptual y jurídica al término acoso laboral. Anales de Derecho, 29, 224-245.

Argüello-López, G. M., Uribe-Bermúdez, J. M., \& ValdiviesoGuerrero, M. (2017). Relación entre capacitación y actitud hacia los riesgos laborales en el sector construcción del área metropolitana de Bucaramanga. I+D Revista de Investigaciones, 9(1), 14-26. https://doi. org/10.33304/revinv.v09n1-2017002

Brunet, L. (1987). El clima de trabajo en las organizaciones: Definición, diagnóstico y consecuencias (Editorial).

Charria, V. H., Sarsosa, K. V., \& Arenas, F. (2011). Factores de riesgo psicosocial laboral: métodos e instrumentos de evaluación. Revista Facultad Nacional de Salud Pública, 29(47), 380-391.

Comisión Europea. (2002). Guía sobre el estrés relacionado con el trabajo. ¿La "sal de la vida" o "el beso de muerte"? Luxemburgo: Oficina de Publicaciones Oficiales de las Comunidades Europeas.

Comité Mixto OIT-OMS sobre Medicina del Trabajo. (1984). Factores psicosociales en el trabajo: naturaleza, incidencia y prevención. Ginebra.

Cornell, F. (1955). Socially perceptive administration. El Phi Delta Kappan, 36(6), 219-223.

Cortés-Reyes, É., Rubio-Romero, J., \& Gaitán-Duarte, H. (2010). Métodos estadísticos de evaluación de la concordancia y la reproducibilidad de pruebas diagnósticas. Revista Colombiana de Obstetricia y Ginecología, 61(3), 247-255.

COTAXI. (2018). Nuestra empresa. Recuperado de http:// www.cotaxi.com.co/empresa/empresa

Espinosa, J. C., \& Romero, L. (2002). Cuestionario de factores psicosociales en el trabajo: Manual. Bogotá: HL BIO SA.

Fellner, D. J., \& Sulzer-Azaroff, B. (1984). A behavioral analysis of goal setting. Journal of Organizational Behavior Management, 6(1), 33-51.

Gibson, J. L., Donnelly, J. H., \& Ivancevich, J. M. (1991). Organizations: behaviour, structure, processes.

Gobierno de Colombia. (2006). Ley 1010 de 2006. Recuperado de https://www.funcionpublica.gov.co/ eva/gestornormativo/norma.php?i=18843

Gómez, M. C. S. (2015). La dicotomía cualitativo-cuantitativo: posibilidades de integración y diseños mixtos. Campo Abierto. Revista de Educación, 1(1), 11-30.
Hall, R., \& Deras Quiñones, A. (1996). Organizaciones: Estructura, Procesos y Resultados (Prentice-H).

ICONTEC. Guía para la identificación de los peligros y la valoración de los riesgos en seguridad y salud ocupacional, Pub. L. No. GTC 45 (2010). Colombia: Guía Técnica Colombiana.

Jiménez, B. M., \& León, C. B. (2010). Factores y riesgos psicosociales, formas, consecuencias, medidas y buenas prácticas. Universidad Autónoma de Madrid.

Leaño, A. (2018). Relevancia de las Mipymes en el área metropolitana de Bucaramanga. I+D Revista de Investigaciones, 12(2), 70-76.https://doi.org/10.33304/ revinv.v12n2-2018008

Likert, R. (1968). Tipos de Clima Organizacional: El factor humano de la empresa (Bilbao: Ed). Bilbao.

Litwin, G., \& Stringer, R. A. (1968). Motivational and organizational climate. Harvard University.

Martínez, G., Cortés, M. E., \& Pérez, A. D. (2016). Metodología para el análisis de correlación y concordancia en equipos de mediciones similares. Revista Universidad y Sociedad, 8(4), 65-70.

Méndez, C. E. (2006). Clima Organizacional en Colombia EI IMCOC: un método de análisis para su intervensión. Centro editorial Universidad del Rosario (Vol. 4). Bogotá.

OIT. (2003). Repertorio de recomendaciones prácticas sobre la violencia en el lugar de trabajo en el sector de los servicios y medidas para combatir este fenómeno. Ginebra.

Orellana, B. J., \& Portalanza, C. A. (2014). Influencia del liderazgo sobre el clima organizacional. Influencia del liderazgo sobre el clima organizacional, 5(11), 117-125.

Palacios, A. (1998). Diagnóstico organizativo. Análisis de los modelos metodológicos (Editorial).

Reinoso Alarcón, H., \& Araneda Cea, B. G. (2007). Diseño y validacion de un modelo de medicion del clima organizacional basado en percepciones y expectativas. Revista Ingeniería Industrial, 6(1), 5.

Rodríguez, D. (2001). Diagnóstico Organizacional. (Alfaomega). México. D. F.

Rosero, A. C., \& Álvarez, C. C. (2012). Riesgos psicosociales intralaborales en docencia. Revista lberoamericana de Psicología: Ciencia y Tecnología, 5(2), 95-106.

Salazar Estrada, J. G., Guerrero Pupo, J. C., Machado Rodríguez, Y. B., \& Cañedo Andalia, R. (2009). Clima y cultura organizacional: dos componentes esenciales en la productividad laboral. Acimed, 20(4), 67-75.

Seisdedos, N. (1996). El clima laboral y su medida. Psicología Del Trabajo y de Las Organizaciones, (2).

Torrecilla, O. D. (2005). Clima organizacional y su relación con la productividad labora. Facultad de ciencias Políticas y sociales.

Uribe, A. (2013). Factores psicosociales en las organizaciones. Universidad Pontificia Bolivariana. 